\title{
„Er hat all die Jahre geschwiegen": Zwischen Tabu und Tabubruch in Memoiren von Gulag-Opfern aus der DDR
}

Im Roman Das Vertrauen (1968) lässt Anna Seghers den Werkleiter Ulsperger erst nach dem Volksaufstand am 17. Juni 1953 in einem privaten Gespräch mit dem Parteisekretär Richard Hagen, mit dem er über zwei Jahre lang zusammen gearbeitet hat, flüchtig erwähnen, dass er während des Exils in der Sowjetunion „schuldlos eingesperrt wurde“ (1968, 439). Noch unter Schock zeigt Hagen, ein ehemaliger Interbrigadist im Spanischen Bürgerkrieg 1936-1939, für solch ein Verschweigen des erlittenen Unrechts im gelobten Land des Sozialismus vollstes Verständnis. Denn darin erkennt er ein musterhaftes Opfer, eine Art Märtyrertum im Sinne des von Siegfried Kracauer für die abgehärteten „Träger der revolutionären Bewegung“ (1932, 5) in Seghers’ Die Gefährten (1932) geprägten Begriffs: „Sie werden verfolgt, gemartert, in die Gefängnisse geworfen; sie führen auch in der Emigration das Dasein von Kämpfern. Wer aktiv für die Sache der Revolution eintritt, nimmt in der Regel nicht sich selber wichtig, sondern die Sache“ (Kracauer 1932, 5). ${ }^{1}$ Zumal wenn der sozialistische Aufbau in der DDR durch die Information über die Verbrechen zur Zeit Stalins gefährdet sein könnte - eine Position, in der der Parteisekretär Richard Hagen mit der offiziellen Linie des SED-Regimes übereinstimmt. ${ }^{2}$ Damit „die Parteiideologen der SED ein homogenes Geschichtsbild [formen konnten], aus dem sich eine Verpflichtung für die Gestaltung einer besseren Zukunft, sprich der sozialistische Aufbau, ableiten ließ“ (Jung 2008, 121), waren autobiografische Zeugnisse von Opfern der stalinistischen Repression in der DDR tabuisiert, und nicht selten war das Tabu ein selbst auferlegtes.

Jene Autoren und Autorinnen, die im Kommunismus unbeirrt ihre ideologische Heimat sahen, verurteilten die Vorgehensweise solcher als Renegaten (Rohrwasser 1991, 1996) stigmatisierten Kommunisten wie Margarete Buber-Neumann,

1 Der religiös-theologische Begriff des Märtyrers oder ,Blutzeugen` wird somit säkularisiert und auf die kommunistische Bewegung übertragen.

2 Solch eine ,Billigung، der Verbrechen in Stalins Sowjetunion soll Anne Applebaum zufolge auch im Westen denkbar gewesen sein: „It is not only the far Left, and not only Western communists, who were tempted to make excuses for Stalin's crimes that they would never have made for Hitler's. Communist ideals - social justice, equality for all - are simply far more attractive to most in the West than the Nazi advocacy of racism and the triumph of the strong over the weak" $(2004,8)$. 
Wolfgang und Susanne Leonhard, Gustav Regler oder Arthur Koestler. ${ }^{3}$ Ihnen gegenüber waren sie bereit, ihre Erfahrung des Unrechts und der Gewalt in der Sowjetunion zu verschweigen, sie zu verdrängen (Emmerich 1996, 326-327). Wenn sie aber trotzdem in der Überzeugung, zur Erschließung der Parteigeschichte beizutragen, ihre Selbstzeugnisse zum Beispiel für das Zentrale Parteiarchiv der SED beim Institut für Marxismus Leninismus bereitstellten, wurden diese „mit einem Sperrvermerk versehen“ (Erler 2001, 188). So wurden die Memoiren Totgesagt: Erinnerungen von Trude Richter (d.i. Erna Barnick, Magdeburg, 19. November 1899 - Leipzig, 4. Januar 1989) und diejenigen von Helmut Damerius (Berlin, 16. Dezember 1905 - 29. September 1985) mit dem Titel Unter falscher Anschuldigung: 18 Jahre in Taiga und Steppe, die jeweils Anfang der 1960er und der 1980er Jahre fertig geschrieben worden waren, erst 1990 publiziert. ${ }^{4}$

Dass die 1981 begonnene Arbeit des Historikers Wolfgang Ruge (Berlin, 1. November 1917 - Potsdam, 26. Dezember 2006) an seiner Autobiografie Gelobtes Land: Meine Jahre in Stalins Sowjetunion (2012), nur langsam vorankam, zwischen 1989 und 1998 unterbrochen und erst 2002 vollendet war, ${ }^{5}$ mag ferner die ideo-

3 Siehe Als Gefangene bei Hitler und Stalin: Eine Welt im Dunkel (München 1949) von Margarete Buber-Neumann, Die Revolution entlässt ihre Kinder (Köln und Berlin 1955) von Wolfgang Leonhard, Gestohlenes Leben: Schicksal einer politischen Emigrantin in der Sowjetunion (Frankfurt a. M. 1956) von Susanne Leonhard, Das Ohr des Malchus: Eine Lebensgeschichte (Köln und Berlin, 1958) von Gustav Regler, Ein Gott der keiner war (Konstanz u.a., 1950) von Arthur Koestler. 41972 war das erste Buch von Richters Manuskript, das ihre Erinnerungen an den Bund proletarisch-revolutionärer Schriftsteller (BPRS) zum Inhalt hat, mit dem Titel Die Plakette. Vom großen und vom kleinen Werden im Mitteldeutschen Verlag (Halle) erschienen. Die ersten drei Abschnitte aus dem zweiten Buch, Tod und Auferstehung, in dem Richters Haft- und Gulag-Erfahrung sowie ihre Verbannung in der Sowjetunion dargelegt werden, wurden unter dem Titel Station Kilometer sieben in der Zeitschrift Sinn und Form (Richter 1988, 498-528) veröffentlicht. 1977 publizierte der Henschelverlag (Berlin-Ost) Damerius' Erinnerungen an die Theatergruppe Kolonne Links mit dem Titel Über zehn Meere zum Mittelpunkt der Welt. Über die Jahre, die Damerius in Gefängnissen, im Gulag und in der Verbannung in der Sowjetunion verbrachte, wurde von Werner Mittenzwei unter dem Titel Helmut Damerius: Porträt eines Mannes vor dem Hintergrund des Jahrhunderts in Sinn und Form (Mittenzwei 1987) ausführlich informiert. Neun Kapitel aus Damerius' Unter falscher Anschuldigung: 18 Jahre in Taiga und Steppe wurden in Sinn und Form (Mittenzwei 1989) abgedruckt.

5 Der Herausgeber Eugen Ruge zitiert eine Fußnote im Originalmanuskript: „Ich [d.i. Wolfgang Ruge] stütze mich hier auf Notizen und z. T. schon recht ausführliche Aufzeichnungen, die ich in großen Abständen seit den sechziger Jahren angefertigt habe“ (2012, 449). Dazu informiert Eugen Ruge: „Diese Aufzeichnungen sind verloren“ (2012, 449). Nach Vollendung der Arbeit wurde das Buch 2003 unter dem Titel: Berlin - Moskau - Sosswa: Stationen einer Emigration (Bonn: Pahl-Rugenstein) veröffentlicht. Dieser Text wurde 2012 von Eugen Ruge überarbeitet und unter dem Titel Gelobtes Land: Meine Jahre in Stalins Sowjetunion herausgegeben. Bei der 
logische Herausforderung belegen, die die Auseinandersetzung mit dem größten Unrecht in der Sowjetunion für überzeugte Kommunisten bedeutet haben muss. In dieser Hinsicht rechtfertigte Trude Richter, die ihre Memoiren als „eine persönliche Rechenschaft über die Bewältigung meiner Vergangenheit“ (1990, 312) sah, die historische Notwendigkeit solcher Erinnerungen im DDR-Kontext mit folgenden Worten, ausgesprochen im multilateralen Kolloquium Memoirenliteratur und Geschichtsbewußtsein (Berlin-Ost, 3.-5. Februar 1981):

Heute ist es eine [...] ABC-Weisheit, daß es bei der Bewältigung der riesigen Schwierigkeiten garnicht [sic] ohne Irrtümer, Fehler, Versäumnisse, Ungerechtigkeiten und ähnliches abgehen kann. Es muß ausgesprochen werden, dargestellt, sonst entstünde eine rosige Schönfärberei, die nicht der Größe und der Kompliziertheit unserer historischen Umbruchsperiode gerecht werden könne [...], jedoch nicht ohne Einbeziehung der riesigen positiven Errungenschaften. (zitiert nach Jung 2008, 134)

Ziel dieses Beitrags ist es, am Beispiel der Lebenserinnerungen der Kommunisten Richter, Damerius und Ruge die konkreten Konturen eines Opfernarrativs zu beleuchten, das sich - obgleich in verschiedenem Ausmaß - in einer im Kern undifferenzierten, zwischen ,Schönfärberei“ und Wahrheitsgehalt schwankenden Artikulationsart manifestiert. Der dementsprechend problematisch-kompromittierende Zusammenprall von Verschweigen bzw. Verschleierung - des revolutionären Märtyrers oder des „Heldenopfer[s]“ (Assmann 2016, 146) - und Verbitterung bzw. Anklage - des Gulag-Überlebenden oder des „Leidensopfer[s]“ (Assmann 2016, 146) - soll dabei ermittelt werden. Nicht zuletzt soll dadurch belegt werden, „dass der Opferbegriff schillert und zwischen gegensätzlichen Haltungen wie Aktivität und Passivität, Stolz und Apathie, Sinngebung und Verzweiflung oszilliert“ (Assmann 2016, 146). Eine kurze biografische Notiz sei zur Erläuterung des jeweiligen bitteren Loses vorangestellt.

\section{Leben und Überleben, Schweigen und Schreiben}

Trude Richter, 1. Sekretär des Bundes proletarisch-revolutionärer Schriftsteller, Helmut Damerius, Leiter der Agit.-Prop.-Gruppe Kolonne Links, und Wolfgang Ruge, junger Pionier und Mitglied des Sozialistischen Schutzbundes und des Kommunistischen Jugendverbandes Deutschlands, emigrierten jeweils Anfang

Überarbeitung wurde der Text „gekürzt und einem kritischen Vergleich mit späteren Fassungen unterzogen“ (E. Ruge 2012, 455). 
und Mitte der 1930er Jahre in die Sowjetunion. Im Rahmen der Jeschowschtschina, der Terrorkampagne 1936-1938 unter Nikolai Jeschow, Leiter des Staatssicherheitsdienstes, wurden Richter und Damerius verhaftet - sie wegen „konterrevolutionärer trotzkistischer Tätigkeit“ (Richter 1990, 295), er unter dem Verdacht der Spionage (Damerius 1990, 50) -, um dann in den Gulag geschickt zu werden. Richter kam ins Lagergebiet von Kolyma, Damerius ins Lagergebiet Solikamsk im Nordural. Ruge wurde nach dem Überfall der deutschen Wehrmacht auf die Sowjetunion im Juni 1941 im September jenes Jahres als verdächtiger Deutscher aus Moskau nach Kasachstan „ausgewiesen“ (Ruge 2012, 120). Er arbeitete in einem Kolchos im Verwaltungsbezirk Ossakarowka bis Anfang 1942, dann wurde er als Arbeitsarmist im Lagergebiet Soswa im Nordural interniert.

Richter wurde 1946 entlassen und 1949 wieder verhaftet und nach Ustj-Omtschug verbannt („Wieder Paragraf 58“, „meine konterrevolutionäre Tätigkeit“, Richter 1990, 391 [Hervorhebung im Original]), um zur Bergbauverwaltung zugewiesen zu werden. Damerius wurde nach Verbüßung der Strafe erneut verurteilt (wegen „konterrevolutionärer Agitation“, Damerius 1990, 226), aber 1946, nach Revision des Prozesses, freigesprochen (Damerius 1990, 236). Er musste noch ein Jahr im Lager bleiben, dann wurde er in die Verbannung geschickt. Ruge blieb nach 1945 als Zeichner und später Ingenieur im Projektierungsbüro eines Holzbetriebs und nahm ein Fernstudium auf. 1955 wurde seine Verbannung aufgehoben und er kehrte nach Deutschland, in die DDR, zurück. Auch Richter und Damerius wurden Mitte der 1950er Jahre rehabilitiert und zogen ebenfalls in die DDR. Richter ließ zwanzig, Damerius achtzehn und Ruge fünfzehn Jahre ungerechter Strafe hinter sich. ${ }^{6}$

In der DDR waren sie alle schnellstens integriert: Richter wurde an das Leipziger Johannes R. Becher Institut für Literatur gerufen, Damerius wurde künstlerischer Leiter der Konzert- und Gastspieldirektion in Berlin, Ruge Professor der Akademie der Wissenschaften. Dafür waren sie verpflichtet, über ihre Leidenswege in der Sowjetunion zu schweigen, eine Verpflichtung, die von Johannes R. Becher als „eine unausgesprochene gesellschaftliche Regel“ $(1988,546)$ erkannt wurde. Eben diese Regel wurde sogleich von Richter und Ruge verinnerlicht, als erstere von Becher empfangen wurde, der damals Kulturminister der DDR war, und letzterer von Karl Schirdewan, Mitglied des Politbüros, der als der zweite

6 Ruge war Mitte zwanzig, als er aus Moskau zwangsevakuiert wurde, Ende dreißig kam er frei. Richter und Damerius waren Mitte dreißig, als sie verhaftet wurden, jeweils Mitte und Anfang fünfzig kamen sie frei. Dazu schreibt Damerius: „achtzehn Jahre, die besten Jahre, die ein Mann besitzt. Die mich verhaftende Instanz merkte ihren Irrtum erst siebzehn Jahre später“ (1990, 11 [Hervorhebung im Original]). 
Mann nach Walter Ulbricht galt. „Ergriffen lauschte er [Becher] meinem Bericht. Ich sah, wie sehr er litt unter allem, was geschehen war, und überzeugte mich, daß er uns nie verdächtigt und auch nicht vergessen hatte“ $(1990,456)$, schreibt Richter. „Obwohl er [Schirdewan] meine Odyssee in der Sowjetunion mit keinem Wort erwähnt, spüre ich, dass er bemüht ist, das mir widerfahrene Unrecht wiedergutzumachen“ (2012, 438), bemerkt Ruge schlicht. Nur Damerius artikuliert eine Klage, während er aber auch das Mitgefühl und die Hilfsbereitschaft der Menschen in Moskau zu ihm, dem Rehabilitierten, zu schätzen weiß: „Ich wurde von allen als eine Art Held gefeiert, der aus einem anderen Kriege lebendig zurückgekehrt war. [...] Es wunderte mich, niemand, auch keiner aus dem Freundeskreis, fragte, warum, wieso, weshalb. Wer hätte die Fragen auch beantworten können“ (1990, 322, 329). ${ }^{7}$

Trotz der Schweigepflicht hatte Richter schon 1956 mit „Vorarbeiten für ihre Autobiografie“ (Jung 2008, 136) begonnen, die 1962 fast vollständig abgeschlossen war. Dabei musste Richter auf ihren „wohltrainierten Gedächtnisfilter“ (1990, 452) vertrauen, denn sie hatte ihre in der Sowjetunion niedergeschriebenen „Manuskripte und Tagebücher [...] der vernichtenden Kritik des Herdfeuers“ (1990, 452) übergeben - „ich wollte mir das Hineinwachsen in die deutsche Gegenwart nicht erschweren durch philosophisches Gepäck aus einer überwundenen Etappe“ (1990, 452) - führt sie als Rechtfertigung dafür an. Ob der heutige Leser bei einem solchem „Filter“ etwa an eine Art „Selbstzensur“ denken soll, sei dahingestellt. Allerdings musste die Publikation über 35 Jahre lang warten. ${ }^{8}$ Damerius' Manuskript war im Februar 1982 fertig. Ein Exemplar übergab der Autor Werner Mittenzwei, ein weiteres Buch kam im Dezember 1983 in das Archiv der Akademie der Wissenschaften der DDR (Mittenzwei 1990, 365). Über das schwierige Fortkommen des Memoirenschreibens von Wolfgang Ruge überlegt der Herausgeber Eugen Ruge, Sohn des Autors:

7 Konkret gegenüber den Genossen des Nationalkomitees Freies Deutschland äußert Damerius seine Enttäuschung: „Ihre unschuldig verhafteten Genossen hatten sie vergessen, oder sie hatten nicht die Zivilcourage gehabt, sich an sie zu erinnern und sich für sie einzusetzen“ (1990, 260). Trotzdem fragt er sich auch: „Hatte ich vor 1938, als ich noch in der Freiheit war, gefragt, wo sind die Verhafteten geblieben? Versteckte ich mich damals nicht genau wie die, die es noch nicht getroffen hatte, hinter dem unerschütterlichen Glauben an die Partei und an die Unfehlbarkeit Stalins?“ (1990, 289).

8 In der DDR hat Richter zweimal, 1965 und 1973, versucht, ihre Memoiren zu veröffentlichen, ohne Erfolg: Das Manuskript blieb im Lager des Zentralen Parteiarchivs der SED. Darüber informiert Jung (2008, 134-137) ausführlich. 
[Es] bleibt [...] erstaunlich, dass ein Mann [d.i. Wolfgang Ruge], der in 26 Jahren seines aktiven Berufslebens über 800 Publikationen zusammengebracht hat (darunter viele Bücher und umfangreiche Beiträge zu Sammelbänden), im Verlauf von sieben oder sogar acht Jahren nicht über die erste Hälfte des geplanten Projekts hinauskommt. (E. Ruge 2012, 450)

Gewiss hat Wolfgang Ruge „zeitlebens nach einer angemessenen Form für die Verarbeitung seines Traumas gesucht“ (E. Ruge 2012, 449). „Ganz offensichtlich“ sind es zudem Eugen Ruge zufolge „die Ereignisse im November 1989“ (2012, 450) welche schließlich eine Unterbrechung von neun Jahren verursachen.

Zumindest Richter und Damerius sollen der Meinung gewesen sein, erst durch die schonungslose Schilderung des erfahrenen Unrechts würden ihre Jugendziele „weiter verfolgbar und erstrebenswert“ (Mittenzwei 1990, 364). Zudem sollen sie im Verfassen ihrer Memoiren eine Verpflichtung gegenüber denen gesehen haben, die den Gulag nicht überlebt hatten (Mittenzwei 1990, 364). Diese Gründe mögen als Motivationsfaktoren angesehen werden, welche sie schließlich zum Versuch, „die Geschichte in ihren Widersprüchen und mit ihren tatsächlichen Menschenschicksalen vorzuführen“ (Mittenzwei 1990, 364-365), veranlasst haben. Oder, wie es von Eugen Ruge zusammengefasst wird, indem er sich konkret auf die Erinnerungen seines Vaters bezieht: „Es ist nicht nur ein beeindruckendes Zeugnis menschlicher Leidens- und Lebensfähigkeit, es ist zugleich ein außergewöhnliches Zeitdokument“ (2012, 455).

\section{Ein (selbst auferlegtes) Tabu}

Das Tabu, mit dem in der DDR Gefängnis- und Gulag-Erfahrung in der Sowjetunion belegt ist, bedingt vor allem in Richters Memoiren eine „Gelassenheit gegenüber dem eigenen Schicksal bei allem Schmerz, [...] [die] für die meisten Leser schwer nachvollziehbar sein“ (Scheer 1990) mag. Ihre „Tragödie - de[n] Versuch, ein Leben nach dem Tode zu führen, d.h. Mensch zu bleiben auch unter den widrigsten Umständen“ (1990, 290) - bezeichnet Richter zum Beispiel als „eine optimistische“ (1990, 290). Veranschaulicht sei hier zunächst der positive Ton, der auch thematisch verwandte Passagen der Erinnerungen von Damerius und Ruge durchzieht, denn erst dadurch lässt sich die Wirkung des selbst auferlegten Tabus in seiner ganzen Tragweite dokumentieren.

Zuallererst freut sich Richter darüber, dass sie im Moskauer Butyrka-Gefängnis zum ersten Mal in ihrem Leben „Zeit, endlos Zeit“ (1990, 292) hat, um Gymnastik zu treiben und „täglich mehrere Stunden mit gewinnbringender Lektüre aus[zu]füllen“ $(1990,292)$ oder auch um Interessenten freiwillig Fremdsprachenunterricht zu erteilen. In demselben Sinn schreibt Damerius über den Lese- 
„Luxus“ im Gefängnis: „[E]inmal in der Woche konnte man ein Buch aus der Gefängnisbibliothek ausleihen. [...] [E]s gab sogar Bücher in deutscher Sprache“ $(1990,47)$. Des Weiteren bemerkt er, dass er darin das „Russischlernen“ $(1990,48)$ fortsetzen konnte.

Ferner feiert Richter die bejahende Haltung der Gefangenen zur sozio-politischen Realität in der Sowjetunion: „[K]einer [der mitgefangenen Frauen] kam es in den Kopf, die Errungenschaften der Sowjetmacht in Zweifel zu ziehen. Und alle waren erfüllt von Optimismus für die Zukunft ihres Landes“ (1990, 294). Wie Richter nicht daran zweifelt, dass sich ihr Schicksal wie das ihrer Leidensgefährten „eines Tages zum Guten wenden“ $(1990,301)$ wird, so hält Damerius auch an seinem kommunistischen Glauben und an Stalin fest. Im Lager angekommen, beteuert er: „[I]ch war voller Hoffnung, von hier aus mein Recht zu finden. Ich würde an Stalin schreiben, alles würde sich aufklären, und die Gerechtigkeit würde wieder hergestellt werden. Auch hier würde ich mich wie ein Kommunist verhalten und gute Arbeit zum Nutzen der Sowjetmacht leisten“ (1990, 72). Insgesamt hat Damerius siebzehn Eingaben an Stalin geschrieben, die alle unbeantwortet geblieben sind (Damerius 1990, 53), was Michael Rohrwasser nicht nur im Sinne einer loyalistisch-gläubigen Haltung interpretiert:

In diesen Briefen kann sich die letzte Loyalität der alten Parteigenossen verbergen, aber auch der Glaube an „Väterchen Stalin, der von allem nichts wußte“ - eine verwandte Redewendung klingt im Ohr. [...] Zugleich wird mit dem Adressaten Stalin die besondere Bedeutung (und Gefährlichkeit) der eigenen Person hervorgehoben[.] [...] Eine Antwort [...] konnte es nicht geben[.] (Rohrwasser 1991, 274)

Ein weiteres Thema sind Verhöre und die Anwendung von physischer Gewalt, die von Richter einfach verschwiegen wird. Das einzige in ihren Memoiren angeführte Verhör soll eine Viertelstunde gedauert haben (1990, 295), ein anderes Mal sei sie „von einem höheren Offizier sehr zuvorkommend empfangen“ (1990, 295) worden und habe sich sogar auf dessen Diwan ausruhen dürfen, ohne dass es dann zum Verhör gekommen sei. Damerius relativiert die Gewaltausübung oder registriert sie aus der Distanz. Er habe zum Beispiel beim „Baden“ einen nackten Mann gesehen, der in einem anderen Gefängnis verhört worden war und „der fast am ganzen Körper grün und blau und angeschwollen war und der sofort umfiel“ (1990, 28). Ein weiterer Häftling sei vom Untersuchungsrichter mit einem Lineal auf die Oberschenkel geschlagen worden. Sonst schreibt Damerius: „[E]rst nach 1938 [wurde] zu aktiveren Methoden beim Verhör gegriffen“ (1990, 35).

Später, beim Transport und auch im Lager, wird die imposante Schönheit der sibirischen Landschaft gepriesen. Richter beschreibt zum Beispiel die Fahrt durch die Kolyma-Region ,[i]m offenen LKW“ $(1990,305)$ in einem feierlichen Ton: „Auf funkelnagelneuen Chausseen rollten wir durch die sommerliche Taiga. Uns ging 
das Herz auf bei ihrem Anblick. Freudig begrüßten wir eine Reifenpanne, die uns eine Rast im Walde und die Bekanntschaft mit seinem Beerenreichtum verschaffte“ (1990, 305). Problematischer wird es nur, als Richter im Wald arbeiten und eine von Pferden getriebene Fuhre durch die unwegsame verschneite Taiga fahren muss, doch auch da findet sich eine hilfsbereite Hand (1990, 323-326). Damerius, der den Reiz des urwüchsigen sibirischen Waldes und das atemberaubende Phänomen der weißen Nächte und des Polarlichts erkennt, schildert allerdings auch sein Leiden als Waldarbeiter: „Als ich nun unfreiwillig durch einen solchen Wald marschierte, war mein Gefühl für seine Schönheit stark gedämpft. [...] Und der schöne Wald, die Taiga, erschien mir dunkel, unfreundlich drohend und Angst einflößend“ (1990, 69 [Hervorhebung im Original]). Auch Ruge, der in seinem Buch zunächst mehrere Absätze dem Preis der Steppenlandschaft in Karaganda widmet (2012, 139-140), ist später, als Arbeitsarmist im Nordurallager, nicht mehr imstande, die Schönheit der Taiga zu genießen: „Die Sonne steht jetzt knapp über dem Horizont, der Himmel schimmert in durchsichtigem Grau, kleine weiß gepuderte Tannen recken ihre Spitzen in die Höhe. Eigentlich entbehrt auch diese Landschaft nicht gewisser Reize. Wenn nur der Magen nicht so knurren würde!“ (2012, 174), ist in seinen Memoiren zu lesen.

Zur schweren körperlichen Arbeit im Lager äußert sich Richter in einem ganz positiven Ton. Für sie sei diese Arbeit nämlich bereichernd und befriedigend, zumal: „Mir fehlte jegliche Kenntnis im Umgang mit solch einfachen Werkzeugen wie Messer, Säge, Sense, Hacke, Beil, Harke. Nie hatte ich die Bekanntschaft gemacht mit dem treuen Helfer der Menschen seit Jahrtausenden, dem Pferde, und besaß keinen Begriff von den Anstrengungen der Bauern, der Holzfäller“ (1990, 329-330). Durch die Arbeit will Richter sich folglich „in das wirtschaftliche Leben des Gebiets einbezogen und damit letzten Endes doch für den sozialistischen Aufbau tätig“ (1990, 333) wissen. Schließlich habe sie dadurch den „Zusammenhang zwischen Theorie und Praxis“ (1990, 82) des Sozialismus erfahren können. Damerius, der schwerste Waldarbeit leisten muss, findet erst durch die vorübergehende Betätigung in einer „Kultbrigade“ und durch das Theaterspielen „eine Aufgabe im Rahmen des Lagerlebens und damit wieder Anschluß an das Leben überhaupt“ $(1990,136)$. Später freut er sich über seine Mitarbeit an der „Kultur- und Erziehungsabteilung“ $(1990,157)$, wo er die einfachen Tafeln der Lagerwandzeitung herstellt, auf denen die täglichen Arbeitsergebnisse, die Verstöße gegen die Disziplin und alle Planrückstände verzeichnet wurden. Das ist für Damerius eine Arbeit, die nicht nur angenehmer ist, als im verschneiten Wald Bäume zu fällen, sondern auch eine, die er „für gut und politisch richtig“ (1990, 158) hält, und „die den Gepflogenheiten in der Sowjetunion entspr[i]ch[t]“ (1990, 158-159). Ruge, auch als Holzarbeiter eingestellt, empfindet seinen zeitweiligen Einsatz als Brigadier bei der Heumahd als positive Wendung, denn endlich kann 
er sich da satt essen: „Ich sichere den Frauen ihre Brotkanten, füttere meine Leute bestens durch, zweige der Obrigkeit ihre Schober ab. Allmählich kehre ich zu einem menschenähnlichen Leben zurück“ $(2012,291)$.

Nach überstandener Haft und Verbannung bemüht sich Trude Richter darum, das Lager in einem positiven Licht darzustellen. Als ein von ihr angezeigter Dieb zu achtzehn Jahren Arbeitslager verurteilt wird, bemerkt sie zum Beispiel, „daß [...] diese Arbeits- und Besserungsinstitution gründlich umorganisiert“ worden sei, und dass ,,jeder Kriminelle [...] Schulungskurse aller Art absolvieren konnte“ (1990, 433). Überdies betont Richter ihre Freude, „heil geblieben“ (1990, 424) und sogar entlohnt worden zu sein. ,[W]ie jeder, der aus dem fernen Osten kam, [war ich in Moskau 1956] recht wohlhabend“ (1990, 452), merkt sie zufrieden an. Sie kann sich zum Beispiel sämtliche Einkäufe und einen Aufenthalt in einem Sanatorium auf der Krim leisten, wo ihre Gesundheit „[r]estauriert“ (1990, 452) worden sei. Damerius preist seinerseits zunächst die „Gefängnisordnung“: „Bei der Entlassung erhielt ich mein Köfferchen, meine Hosenträger und Schnürsenkel und sogar das restliche Geld, all das, was mir bei meiner Verhaftung [...] abgenommen worden war“ $(1990,53)$. Jahrzehnte später, nach überstandener Lagerhaft, rühmt er sich seiner unbeirrbaren Überzeugung: „[E]s ist nicht gelungen, uns zu Wölfen umzuerziehen. Wir waren Genossen - Kommunisten geblieben“ (1990, 247). Bei Ruge ist erst nach Stalins Tod und erst recht 1956 nach dem XX. Parteitag des Zentralkomitees der KP der Sowjetunion, als die Rückreise nach Deutschland schon bevorsteht, ein optimistischer Kommentar zur politischen Lage im Ostblock zu vernehmen: „[I]ch bin von Zuversicht erfüllt. Ich beginne zu glauben, dass jetzt, genau in diesem Moment, in dem auch mir wieder Flügel zu wachsen scheinen, die internen Machtkämpfe im sowjetischen Politbüro abgeschlossen seien und nun die Phase der Errichtung eines wirklichen Sozialismus beginne“ (2012, 436 [Hervorhebung im Original]).

Für die Unterstützung aus der DDR haben Richter, Damerius und Ruge nur Worte des Dankes und der Anerkennung. Trude Richter erwähnt die Unterstützung von Anna Seghers und von Johannes R. Becher. Das Leipziger Literaturinstitut, in dem sie als Dozentin tätig ist, sieht Richter als „das Ithaka meiner Odyssee“ (1990, 456). Helmut Damerius war seinerseits von Arthur Pieck, dem Sohn des einzigen DDR-Präsidenten, Wilhelm Pieck, zum Umzug in die DDR verholfen worden. Die Reise nennt er eine „Fahrt ins Ungewisse“, wobei aber „die Himmelsrichtung [...] die richtige und von mir lang ersehnte [war]“ (1990, 330). Wolfgang Ruge informiert über den Kontakt zu seiner Mutter Charlotte, Leiterin des Instituts für Literatur und Sprachen an der Akademie für Staat und Recht in Potsdam-Babelsberg, und deren zweiten Ehemann Hans Baumgarten. Sie waren auch diejenigen, die ihn mitsamt Frau und Kind in Berlin in Empfang nahmen und versorgten. 


\section{Trotz alledem: Tabubruch}

Trotz des mehr (bei Richter) oder weniger (bei Damerius und Ruge) offenkundigen positiven Erzähltons bezeugt der Inhalt der drei Memoirenbücher das gewaltige Ausmaß des den Autoren zugefügten Unrechts. Sowohl in Richters Autobiografie als auch in derjenigen von Damerius werden die miserablen Umstände der Haft in den verschiedenen Moskauer Gefängnissen (Lubjanka, Butyrka, Taganka) dargestellt, wobei Damerius deutlich negativere Details wiedergibt. Während bei Richter zum Beispiel lediglich von einer „Massenzelle“ (1990, 291) und von Streitereien unter den Häftlingen $(1990,293)$ zu lesen ist, bebildert Damerius die Vermassung durch die Beschreibung der strengen Stehen-Sitzen-LiegenOrdnung in der Zweimannzelle, in der sechzehn Männer eingesperrt sind (1990, 31-32). Über den „Scheißkübel“ oder „Parascha“ in der Zelle und die sogenannte „Toilettenaktion“9 berichtet nur Damerius (1990, 31, 33), wie auch über die magere Kost: „ein Stück Roggenbrot, ungefähr dreihundert Gramm, und warmes Wasser [...]. Mittags erhielt jeder eine Schüssel Suppe“ (1990, 33-34). Während Damerius über die unerbittlichen Desinfektions- und Badeprozeduren (1990, 26-28) informiert, erwähnt Richter eine einzige Laus, indem sie noch kommentiert: „Das Ungeziefer war dort eine eingeschleppte Rarität“ (1990, 294). Ruge (2012, 124) erfährt seinerseits Vermassung, Gedränge und extremen Platzmangel erst im Transport im schmutzigen Güterwaggon von Moskau in die Steppe.

Das Gefängnisleben kennt aber eine noch raffiniertere Form der Folter: das zermürbende Warten auf das Verhör. Dazu hält Damerius fest: „Die Mitteilung ,vorbereiten zum Verhör‘ erteilte der Schließer am Tage, aber geholt wurde man meistens nachts. Verhören wollte man müde Leute, die sich bis zum Verhör durch Grübeln oder Angst schon selbst zermürbt hatten, die keinen Widerstand leisten konnten“ $(1990,34)$. Sowohl er als auch Richter berichten zudem von der Qual der Ungewissheit über die eigene Zukunft, allein Ruge beteuert: „Die Unwissenheit schützt mich“ (2012, 171). Damerius und Richter bemängeln darüber hinaus die strenge Isolation von der Außenwelt, die Richter mit dem Tod gleichsetzt: „[I]ch war mir klar darüber, daß ich ausgelöscht war für alle anständigen Menschen. Tot für meine Genossen in Moskau, in Berlin, in Paris und wo sie sonst in der Emigration weilten, tot für meine Schüler“ (1990, 312). Für Damerius (1990, 54, 61) bringt die Isolation auch noch den Verlust des Zeitbewusstseins mit sich sowie eine

9 Von Damerius (1990, 33) wie folgt beschrieben: „[J]e sechs Mann [wurden] auf die Toiletten geführt. Es waren Hockklosetts - sechs Löcher auf einem langen Zementsockel, ohne Trennwände und ohne Türen, mit Wasserspülung, die immer lief. Papier gab es nicht, Wasser lief ja. Es gab auch ein Waschbecken, aber zum Waschen war keine Zeit bei dem herrschenden Hochbetrieb.“ 
intellektuelle Rückständigkeit: „Ich hatte in diesen achtzehn Jahren fast nichts gelesen, hatte die politische Entwicklung in der Welt nicht miterleben können, hatte nichts dazu gelernt außer einigen Brocken Ganovenjargon“ (1990, 322).

Fernerhin informieren Richter und Damerius über die „Massenabfertigungen“ ohne Prozess (Richter 1990, 296; Damerius 1990, 50), wobei letzterer etliche Artikel aus der Verfassung der UdSSR von $1936^{10}$ anführt, um seine Erfahrung deutlich als Verstoß gegen gerade jene Artikel zu kategorisieren. Ruge beklagt seinerseits, dass er weder „förmlich verurteilt noch jemals förmlich entlassen worden“ (2012, 308) sei, wodurch nicht nur die Rechtlosigkeit des stalinistischen Regimes bloßgestellt werden soll, sondern auch dessen Willkür. Zudem leiden die überzeugten Kommunisten darunter, dass sie im Land ihrer „Ideale und Träume, im Vaterland der Werktätigen“ (Damerius 1990, 17) in den Gulag geschickt worden sind. In diesem Sinn beteuert Richter, das Schlimmste auf der Welt sei, für einen Feind gehalten zu werden, wenn man in Wirklichkeit „der allerbeste Freund ist“ (1990, 297), und Ruge fragt sich: „Wir waren immer Hitlergegner, auch als alle den Hitler-Stalin-Pakt bejubelt haben. Wir haben uns durchweg loyal verhalten. Warum vertraut man uns nicht? Warum setzt man uns mit den Kulaken gleich, die immer gegen die Sowjetmacht waren?“ (2012, 175 [Hervorhebung im Original]).

Richter, Damerius und Ruge sind sich auch weitgehend darin einig, dass das Lager von Korruption und Betrügerei wimmelte. Richter nennt das dauernde Schachern „Lagermechanismus“ (1990, 299), Ruge und Damerius verwenden für ,jede Art obrigkeitsschädigende[n] Betrug[s]“ (Ruge 2012, 220) das Wort im Lagerjargon: „Tufta“ (Damerius 1990, 147; Ruge 2012, 220), dessen Funktionieren Damerius konkret und detailliert beschreibt (1990, 147-157), aber erst aus der Perspektive der 1980er Jahre als lebensrettende Strategie zu erkennen vermag: „Tufta bewahrte Tausende Gefangene vor Hunger und Hungertod, war also für

10 Unter dem Titel Aus der Verfassung der UdSSR von 1936 zitiert Damerius (1990, 55-56): „Artikel 102 Die Rechtsprechung wird in der UdSSR von dem Obersten Gerichtshof der UdSSR, den Obersten Gerichtshöfen der Unionsrepubliken, den Regions- und Gebietsgerichten, den Gerichten der Autonomen Republiken und Autonomen Gebiete, den Bezirksgerichten, von besonderen Gerichten der UdSSR, die auf Beschluß des Obersten Sowjets der UdSSR bestellt werden[,] und von den Volksgerichten ausgeübt.

Artikel 111 Die Verhandlung ist bei allen Gerichten der UdSSR öffentlich, sofern nicht durch das Gesetz Ausnahmen vorgesehen sind; dem Angeklagten wird das Recht auf Verteidigung gewährleistet. Artikel 127 Den Bürgern der UdSSR wird die Unverletzlichkeit der Person gewährleistet. Niemand kann anders als auf Gerichtsbeschluß oder mit Genehmigung des Staatsanwalts verhaftet werden. Artikel 129 Die UdSSR gewährt Bürgern auswärtiger Staaten, die wegen Verfechtung der Interessen der Werktätigen oder wissenschaftlicher Betätigung oder wegen nationalen Befreiungskampfes verfolgt werden, das Asylrecht.“ 
viele Rettung und Wohltat, ich möchte es, gemessen an den Lagerverhältnissen, ein humanistisches Verbrechen nennen“ $(1990,152)$. Nicht zuletzt durch „Tufta“ kann festgestellt werden, dass „eine Pyramide der sozialen Schichtung innerhalb des Stacheldrahtverhaues [existierte]“ (Richter 1990, 310). Ganz oben stand die „Arbeitsaristokratie“ (Richter 1990, 310), unten das „Lagerproletariat“ (Richter 1990, 311), ${ }^{11}$ wobei Richter auf die ideologisch-politische Inkongruenz solch einer Schichtung aufmerksam macht: „Von Tag zu Tag trat der Widerspruch zwischen dem offiziell verkündeten sozialistischen Grundsatz: ,Jedem nach seinen Leistungen“ und der Praxis mit ihrem Wolfsgesetz unverhüllt hervor“ (1990, 310). Jenes Wolfsgesetz im Gulag wird von Damerius zu der prägnanten Formel zusammengefasst: „Ich esse heute, du morgen, du stirbst heute, ich morgen“ (1990, 109). Des Weiteren denunziert er die unmittelbaren Folgen des „Parasitentums“: „Hunger, Unterernährung, Krankheiten und hohe Sterblichkeit“ (1990, 83), wobei aber nicht nur die „räuberische Lageraristokratie“ $(1990,83)$ an den Pranger gestellt wird, sondern auch die Kriminellen - Gelegenheits- und Berufsverbrecher -, die, wie Richter beklagt, „im Gegensatz zu uns [den Politischen] Volksfreunde genannt [wurden] und [...] sich bei der Lagerleitung großer Nachsicht und Bevorzugung [erfreuten]“ (1990, 299 [Hervorhebung im Original]). Das Billigen von Prostitution (Richter 1990, 298-299) liefert ein recht bizarres Beispiel von solcher Nachsicht, meistens besteht die Bevorzugung jedoch in einer etwas besseren Kost. Denn der extreme Hunger ist das Schlimmste im Gulag, und - neben heilloser Kälte und schwerster körperlicher Arbeit - die Hauptursache für Krankheiten und Tod.

Von sich selbst behauptet Richter zwar, bis vor Kriegsausbruch 1941 „durchaus nicht verhungert“ $(1990,338)$ zu sein, den bittersten Hunger illustriert sie aber am erschütternden Erscheinungsbild ihres Ehemannes Hans Günther, der 1938 im Lager Wladiwostok ums Leben gekommen ist:

[D]a tauchte plötzlich vor mir ein langes Gespenst [d.i. Hans Günther] auf, umschlottert von einer weißen Drillichhose, in einem groben, offenen Hemd, das die Rippen des Brustkorbes sehen ließ. Das Skelett trug eine Hornbrille, und seine Augen blickten mich an aus tiefen Höhlen wie aus einem Totenschädel. (Richter 1990, 302-303)

Was den Hunger anbelangt, sei aus den Memoiren von Damerius folgende Schauder erregende Stelle zitiert: „Verhungernde polkten aus dem gefrorenen Kot die Weizenkörner heraus und aßen sie. Ich habe es gesehen“ (1990, 130). Des Weiteren berichtet Damerius, wie die Lagerinsassen sich von Ratten ernähren, wie ihre darbenden Körper die eigene Substanz aufsaugen, wie tote Gefangene erst

11 Ruge beschreibt und differenziert seinerseits genauestens die verschiedenen Typen innerhalb der Lagergemeinschaft im Unterkapitel „Zuträger, Sägeschleifer, Deputierte“ (2012, 195-206). 
nach einer Woche gemeldet werden, so dass ihre Nachbarn solange deren Brot und deren Suppe - die „Balanda“ $(1990,101)$ - kassieren können, oder auch wie „sich die dahinsiechenden Pferde noch als nützlicher [erweisen] als die dahinsiechenden Menschen. [...] sie wurden geschlachtet [...] und verbesserten ein wenig die Suppe derer, von denen sie zu Tode geschunden worden waren“ (1990, 155; vgl. Ruge 2012, 185). Ruge (2012, 258) bringt der Hungerwahn sogar zum Essen von ungekochtem Fleisch eines rotzkranken Pferdes, das geopfert wurde, dessen Leiche man aber vergeblich $\mathrm{zu}$ verbrennen versucht hatte.

Dass Brot das wichtigste Nahrungsmittel im Gulag ist, belegen Richters Worte: „Ihm verdanke ich meine Gesundheit“ (1990, 308). Jenes Brot sei aber glitschig und bestehe „zu einem Großteil aus Hafer- oder Gerstenkleie“ (2012, 184-185), berichtet Ruge, die tägliche Brotration während der Quarantäne wiege 600 Gramm (2012, 184), später, bei Erfüllung der Arbeitsnorm, 500 (2012, 208), und bei Arrest 300: „zum Leben zu wenig, zum Sterben zu viel“ (2012, 232). Als „Hauptnahrquell“ (Richter 1990, 308) angesehen, sind alle Häftlinge, wie Ruge betont, auf Brot fixiert: „[M]eine Zeitrechnung beschränkt sich auf das Zählen der Stunden bis zur nächsten morgendlichen Brotausgabe“ (Ruge 2012, 207). Zucker, Öl, Fisch oder Grütze bekommen die Lagerinsassen nie (Ruge 2012, 185), neben Brot gibt es nur dünnflüssige Suppe, von Damerius wie folgt beschrieben:

Jahrelang aßen wir Suppe, die nach Petroleum schmeckte; der Kutscher, der die Zisterne Pflanzenöl beförderte, tauschte Wodka gegen einige Liter Öl und, damit das Gewicht stimmte, goß er Petroleum zu. Wasser hätte er nicht nehmen dürfen, das vermischt sich nicht mit Öl oder schwimmt bei Frost als Eis auf dem Öl. (Damerius 1990, 82)12

Infolgedessen ausgemergelt und mit Lumpenkleidung werden die Sträflinge bei extremer Kälte zur Schwerstarbeit gezwungen: Sie werden „bis 35 Grad minus zur Waldarbeit hinausgeführt (bei Wind bis 30)“ (Ruge 2012, 209). ${ }^{13}$ Zudem sind sie dreckig und werden von Läusen und Wanzen, im Sommer auch von Fliegen und von Malaria übertragenden Mücken gepeinigt, bis sie krank werden. Die häufigsten Krankheiten im Gulag sind Pellagra - oder Diarrhöe -, Hungerödeme und Dystrophie. Sonst leiden die Lagerinsassen auch an Erfrierungen, an Heme-

12 Damerius (1990, 87-88) klagt diesbezüglich noch, dass die Suppe auf ihren Inhalt nicht kontrolliert oder geprüft wird.

13 Ruge tadelt zudem die organisatorische Sinnlosigkeit der Lagerpolitik: Dass zum Beispiel ein hochqualifizierter Ingenieur, der sogar in Amerika gearbeitet hat, während des Zweiten Weltkriegs in der Sowjetunion ,wegen eines deutschen Urgroßvaters für vergleichsweise lächerliche Arbeiten ein[ge]spannt [wird] - die Instandhaltung von ein paar klapprigen Lkws und Motorbooten im fernen Ural“ $(2012,198)$. 
ralopie - oder Nachtblindheit -, an Furunkeln und an allerlei Verletzungen, wobei die medizinische Versorgung höchst defizitär ist. Mit folgenden Worten beschreibt Ruge die Mittel des Lagerarztes Dr. Wagner: „Mit seinem Zuspruch, seinen Quarantäneverordnungen und der Entschärfung von Befehlen rettet er mehr Menschen als mit seinen oft unter haarsträubenden Bedingungen durchgeführten Operationen“ (2012, 198). Fatale Unfälle, Selbstverstümmelung, um der Arbeit im Wald zu entfliehen, und schließlich auch Selbstmordfälle werden in allen Gulag-Autobiografien registriert. Sogar Trude Richter soll 1950 bei einer Krise den Freitod gesucht haben, wobei der Strick, mit dem sie sich an dem Deckenbalken habe erhängen wollen, glücklicherweise gerissen sei. Bei Damerius und Ruge scheint dagegen der Überlebenswunsch stets überwogen zu haben. Ruge ermunterte sich mit dem Gedanken: „Zusammenbrechen wird er nicht, da muss er durch, auch das wird ein Ende nehmen“ (2012, 208 [Hervorhebung im Original]). Damerius behauptet seinerseits, er habe sich „fest vorgenommen, das Lager lebend zu verlassen“ (1990, 112), was er aufgrund seiner kommunistischen Überzeugung argumentiert: „Selbstmord paßte nicht zu meiner lebensbejahenden kommunistischen Weltanschauung“ (1990, 232).

Aus der zeitlichen Distanz, nach überstandener Haft und Verbannung - dem „bitteren Brocken, den ich nie verschlucken würde“ (Richter 1990, 297) -, macht Richter den Personenkult des Stalinismus dafür verantwortlich, dass „die innerparteiliche Demokratie außer Kraft“ $(1990,417)$ gesetzt worden sei und die Sowjetunion sich in einen „riesigen Sarkophag der Rechtlosigkeit“ $(1990,418)$ verwandelt habe. Wolfgang Ruge fasst aus der Perspektive von 1953 die Ansicht der Opfer der Repression und des Gulags im schlichten Satz zusammen: „Der Tod Stalins rettet Russland“ (2012, 419). Zudem sammelt er die wichtigsten Titulierungen für Stalin als „Menschenschinder“ und als „de[n] größte[n] Schurke[n] aller Zeiten“ (2012, 420). Ebenso denkt und empfindet Helmut Damerius, der seinen Glauben an Stalin endlich korrigiert:

So naiv war ich. Heute ist es mir ganz unbegreiflich, wie ich, und nicht nur ich allein, angesichts der ungeheuerlichen Rechtsverletzungen, die uns ein menschenunwürdiges Dasein, dauernd bedroht von Hunger, Krankheit und Tod, aufzwangen, weiter an den großen Schmied unseres Glücks, Stalin, glauben konnte. (Damerius 1990, 244 [Hervorhebung im Original])

Dementsprechend reagiert Damerius, wenn er in der DDR das Lied von Louis Fürnberg singen hört: „Die Partei, die Partei, die hat immer recht...“. ${ }^{14}$ Da muss er ,an Stalin denken, an Berija, an Jeshow, an Jagoda, an den XX. Parteitag der

14 Das 1949 verfasste Lied diente als offizielle Hymne der SED. Liedtext: http://www.hdg.de/lemo/ html/dokumente/JahreDesAufbausInOstUndWest_liedtextSEDLied/index.html (24. Juli 2018). 
KPdSU, an die Untersuchungsrichter in Moskau und an den Bevollmächtigten im Lager“ (1990, 244), und kann „niemals mitsingen, obwohl ich seit 1923 ununterbrochen Mitglied der Partei, bis heute Kommunist bin und mir nicht vorstellen kann, nicht mehr Mitglied der Partei zu sein“ (1990, 244). In diesen Worten mag trotzdem das „Risiko“ des Renegatentums anklingen, worauf Hans Sahl in der Erzählung Schuld (1969) aufmerksam machte: „Es gibt, glaube ich, drei Phasen in der Entwicklung eines Marxisten zum Antimarxisten. In der ersten macht man Stalin, in der zweiten Lenin, in der dritten Marx selber für die Irrtümer des Marxismus verantwortlich“ $(1992,148)$.

\section{4 „auch wenn uns Schmerz und Scham den Nacken beugen“}

Die Lebenserinnerungen von Trude Richter, Helmut Damerius und Wolfgang Ruge erfassen das Phänomen der persönlichen Rechenschaft von überzeugten Kommunisten angesichts des Stalinismus in einer seiner grausamsten Erscheinungen, dem Gulag. Dabei verstoßen die Autoren erstens gegen die eigene Gesinnung, was als Vorbedingung für die von Richter heraufbeschworene ,rosige Schönfärberei“ der eigenen Darstellung gelten mag. Oder, wie Michael Rohrwasser solch eine Position erläutert:

\footnotetext{
Gerade der Terror schien Ausweis moralischer Größe und Überlegenheit; nicht der Zweck sollte die Mittel heiligen, sondern die blutigen Mittel, der heilige Terror bewies die Größe des Ziels. [...] [W]enn Becher nach mehrfachen Suizidversuchen [der letzte im Moskauer Exilantenhotel $L u x]$ der lebensrettenden Sowjetunion dankte, dann geht es nicht einfach um propagandistische Lügen, dann setzt sich das Bild gegen alle Realitäten ins Recht. (Rohrwasser 1996, 64)
}

Zweitens verletzen Richter, Damerius und Ruge das in der DDR bis zuletzt geltende ideologische Tabu der Unfehlbarkeit des sowjetischen Modells, nach dem jede „Kritik [...] als konterrevolutionäre Haltung entlarvt [wurde]“ (Rohrwasser 1991, 34 [Hervorhebung im Original]), was wiederum eine Darstellungsweise bedingt, die sich als unmögliches Gleichgewicht zwischen Verschweigen und/ oder Beschönigen und wahrheitsgetreuer Wiedergabe materialisiert.

In Richters Totgesagt, Damerius' Unter falscher Anschuldigung und Ruges Gelobtes Land mögen entsprechend etliche Details als Lichtpunkte gelten. Im Gefängnis wird gelesen und es kann Russisch gelernt werden, die Anwendung von physischer Gewalt scheint nicht nachweisbar zu sein. Die Gefangenen sollen eine positive Haltung zum Kommunismus und zur Sowjetunion bewahrt haben, 
gepriesen wird darüber hinaus die Schönheit der Steppenlandschaft und der Taiga. Im Gulag soll nicht zuletzt auch für Kultur gesorgt worden sein, für Theater zum Beispiel. Eventuell bietet die Arbeit als Brigadier bei der Heumahd bessere Überlebenschancen als die Waldarbeit. Nach überstandener Lager- und Verbannungszeit verfügen die deutschen kommunistischen Exilanten über etwas Geld und dürfen in die DDR umsiedeln, wo sie schnellstens integriert werden. Trotz aller Beschönigung überwiegen aber die negativen Eindrücke. Die erste Station sind die Moskauer Gefängnisse: die Vermassung und die unerbittlichen Desinfektions- und Badeprozeduren darin, die Ungewissheit über die eigene Zukunft und die Verurteilungen ohne Prozess. Die Umstände im Gulag danach erregen Entsetzen: es herrschen Betrügerei und Parasitentum; der Hunger ist extrem; unmenschliche körperliche Arbeit muss bei der bittersten Kälte im sibirischen Wald geleistet werden. Hohe Sterbezahlen unter den Häftlingen sind die unmittelbaren Folgen von alledem, sowie von Krankheiten, von Unfällen und nicht zuletzt von Suizid. Die letzte Station, die ewige Verbannung, vervollständigt die Isolation von der Außenwelt. Schuld an der Rechtlosigkeit sind schließlich der Stalinismus und seine Terrormaschinerie, Tabu-Themen in der DDR.

Solche Memoiren sind meines Erachtens folglich als ambivalente Opfernarrative - als Beispiele für verschriftlichte Gulag-Erinnerungen zwischen Tabu und Tabubruch - zu lesen, nicht als Erinnerungsmanipulationen, Fälschungen der Realität oder gar Lügen bzw. Selbstlügen abzuwerten. In dieser Hinsicht soll der hier unternommene Versuch einer kontrastiven Erfassung ihres Zeugnischarakters dazu beitragen, die jeweiligen Schreib- und Publikationsmotivationen zu ermitteln. Das scheint mir aus unserer Perspektive zu Beginn des einundzwanzigsten Jahrhunderts relevant $\mathrm{zu}$ sein: erstens angesichts der Tatsache, dass „ein [...] europäischer Umdenkprozess mit Blick auf die Opfer des Stalinismus noch nicht stattgefunden“ (Assmann 2016, 160) hat, was einerseits wohl auf der „Totalitarismus-These“ oder dem „tief verankerte[n] Tabu“ der Einstufung von Stalinismus und Nationalsozialismus als „zwei Varianten desselben Phänomens“ (Assmann 2016, 161) - gegenüber der „Singularitätsthese“ des Holocausts - zurückzuführen sein mag, andererseits aber sicherlich auch am Vorhandensein solcher kontroversen Lebenserinnerungen von Gulag-Überlebenden wie Richter, Damerius und Ruge liegen könnte; und zweitens in Anbetracht der jüngsten „mentalitätsgeschichtliche[n] Wende“ (Assmann 2016, 58), nach der, wie Aleida Assmann festgestellt hat, „[a]n der Stelle rechter und linker Ideologien [...] die Menschenrechte als normative Grundlage für politisches Handeln, moralische Bewertung und historische Sensibilität [traten.]“ $(2016,58)$. Hierzu, und bei Beachtung der unter anderen von Rüdiger Pohl (2007, 118-146) und Michaela Holdenried (2000, 11-14) festgelegten Funktionen des autobiografischen Erinnerns und Schreibens im Allgemeinen - Bildung des Selbstkonzepts, soziale Interak- 
tion, Selbst-Offenbarung, Erfahrungsweitergabe, Enkulturation -, scheint mir der folgende Kommentar von Nina Frieß für das besondere Genre der Memoiren von Gulag-Opfern von besonderem Wert zu sein:

Das Ablegen eines Zeugnisses (auch gegenüber sich selbst) wurde zum wesentlichen Motivationsmoment des Schreibprozesses. [...] Abrechnen ist in diesem Zusammenhang wohl ein zu starkes Wort, da es impliziert, dass in den Texten eine umfassende Auseinandersetzung mit der Stalinzeit erfolgen würde, in deren Anschluss in der außertextuellen Realität Organisatoren oder Ausführende der Repressionen zur Verantwortung gezogen worden wären. Das war nicht der Fall. Vielmehr wurde die Verantwortung für die Repressionen und Auswirkungen des Personenkults Stalin und einem engen Führungszirkel zugeschrieben [...]. (Frieß 2017, 89 [Hervorhebung im Original])

Die Lebenszeugnisse von Richter, Damerius und Ruge belegen sowohl diese Deutung als auch diejenige von Anne Applebaum, für die:

These [...] were books written both as literature and as testimony: the authors wanted future generations to know what had happened, even if their writings could not be published in their own lifetime. [...] In some cases, memoirists also wanted to transmit their experiences in light of a particular personal narrative. [...] Memoirists were often people with an acute sense of fairness and justice as well. [...] [T] he memoirists sometimes had a moral or didactic intent as well as a purely historical purpose. (Applebaum 2011, XII-XIII)

In Bezug auf die Memoiren von Trude Richter, Helmut Damerius und Wolfgang Ruge sollen schließlich noch die „Wertbindungen“ politischer Erinnerungen, die „stets einen [...] partikularen, gruppenspezifischen Charakter“ (Assmann 2016, 207) haben, beachtet werden, denn sie haben „- ganz im Sinne von Nietzsches Plädoyer für einen eingeschränkten Horizont - die klare Funktion der Handlungsorientierung für die eigenen Ziele, der Stärkung des eigenen Selbstbilds und der Legitimierung der eigenen Taten“ (Assmann 2016, 207).

Wenn auch der Opferbegriff angesichts des fatalen Widerspruchs zwischen kommunistischer Überzeugung und Gulag-Erfahrung „schillern“ und „zwischen gegensätzlichen Haltungen oszillieren“ mag, kann am Ende wohl lediglich von Trude Richter behauptet werden, dass sie „nicht aus der Perspektive des Opfers [berichtet]“ (Scheer 1990). Ihre Haltung ist vielmehr diejenige des Kracauer'schen Märtyrers, den Christa Wolf in ihrem letzten Buch, Stadt der Engel oder The Overcoat of Dr. Freud (2010), zu würdigen weiß, indem sie andere, ebenfalls politische Verse aus der Feder von Louis Fürnberg zitiert. Es sind die folgenden Zeilen aus dem 1953 entstandenen Gedicht „Schwere Stunde“, das erst 1957 in Fürnbergs Nachlass gefunden wurde:

Vielleicht sind wir um eines größren Ziels zum Opfer ausersehn; dann heißt es schweigen, 
auch wenn uns Schmerz und Scham den Nacken beugen

im Anblick dieses Spiels. (Wolf 2010, 86)

\section{Literaturverzeichnis}

Applebaum, Anne. Gulag: A History. London: Penguin, 2004 [2003].

Applebaum, Anne. „Introduction“. Gulag Voices: An Anthology. Hg. Anne Applebaum. New Haven und London: Yale University Press, 2011. VII-XV.

Assmann, Aleida. Das neue Unbehagen an der Erinnerungskultur: Eine Intervention. München:

C. H. Beck, 2016 [2013].

Becher, Johannes R. „Selbstzensur“. Sinn und Form 40.3 (1988): 543-551.

Damerius, Helmut. Unter falscher Anschuldigung: 18 Jahre in Taiga und Steppe. Berlin und Weimar: Aufbau, 1990.

Emmerich, Wolfgang. Kleine Literaturgeschichte der DDR. Leipzig: Kiepenheuer, 1996.

Erler, Peter. „,Mich haben die persönlichen Erlebnisse nicht zum nörgelnden Kleinbürger gemacht': Deutsche GULag-Häftlinge in der DDR“. Vielstimmiges Schweigen: Neue Studien zum DDR-Antifaschismus. Hg. Annette Leo und Peter Reif-Spirek. Berlin: Metropol, 2001. 173-196.

Frieß, Nina. „Inwiefern ist das heute interessant?": Erinnerungen an den stalinistischen Gulag im 21. Jahrhundert. Berlin: Frank \& Timme, 2017.

Holdenried, Michaela. Autobiographie. Stuttgart: Reclam, 2000.

Jung, Christina. Flucht in den Terror: Das sowjetische Exil in Autobiographien deutscher Kommunisten. Frankfurt a. M.: Campus, 2008.

Kracauer, Siegfried. „Eine Märtyrer-Chronik von heute“. Frankfurter Zeitung und Handelsblatt, 13. November 1932, Literaturblatt. 5-6.

Mittenzwei, Werner. „Helmut Damerius: Porträt eines Mannes vor dem Hintergrund des Jahrhunderts“. Sinn und Form 39.4 (1987): 713-739.

Mittenzwei, Werner. „Helmut Damerius: Neun Kapitel über Lebenslauf und Geschichtsverlauf“. Sinn und Form 41.6 (1989): 1287-1314.

Mittenzwei, Werner. „Lebenslauf und Geschichtsverlauf: Skizze zum Porträt eines Mannes vor dem Hintergrund des Jahrhunderts". Unter falscher Anschuldigung: 18 Jahre in Taiga und Steppe. Hg. Helmut Damerius. Berlin und Weimar: Aufbau, 1990. 331-365.

Pohl, Rüdiger. Das autobiographische Gedächtnis: Die Psychologie unserer Lebensgeschichte. Stuttgart: Kohlhammer, 2007.

Richter, Trude. Totgesagt: Erinnerungen. Halle und Leipzig: Mitteldeutscher Verlag, 1990.

Rohrwasser, Michael. Der Stalinismus und die Renegaten: Die Literatur der Exkommunisten. Stuttgart: Metzler, 1991.

Rohrwasser, Michael. „Was haben sie verraten, die Renegaten? Zwölf Thesen zur Faszination des Stalinismus“. Denken im Zwiespalt: Über den Verrat von Intellektuellen im 20. Jahrhundert. Hg. Werner von Bergen und Walter H. Pehle. Frankfurt a. M.: Fischer, 1996. 61-81.

Ruge, Eugen. „Nachwort“. Gelobtes Land: Meine Jahre in Stalins Sowjetunion von Wolfgang Ruge. Hg. Eugen Ruge. Reinbek bei Hamburg: Rowohlt, 2012. 445-456. 
Ruge, Wolfgang. Gelobtes Land: Meine Jahre in Stalins Sowjetunion. Hg. Eugen Ruge. Reinbek bei Hamburg: Rowohlt, 2012.

Sahl, Hans. Der Tod des Akrobaten. Erzählungen. Hamburg und Zürich: Luchterhand, 1992. 143-150.

Scheer, Regine. „Schonungsloser fragen: Totgesagt: Trude Richter erinnert sich an ihr Leben als verfolgte Kommunistin“. DIE ZEIT, 9. März 1990, http://www.zeit.de/1990/11/ schonungsloser-fragen/komplettansicht (29. Dezember 2017).

Seghers, Anna. Das Vertrauen. Berlin und Weimar: Aufbau, 1968.

Wolf, Christa. Stadt der Engel oder The Overcoat of Dr. Freud. Berlin: Suhrkamp, 2010. 
